\title{
A PARASITOLOGICAL VIEW OF NON-CONSTRUCTIBLE SETS
}

\author{
FREDERIK KORTLANDT
}

The genetic code, the primary manifestation of life, and, on the other hand, language, the universal endowment of humanity and its momentous leap from genetics to civilization, are the two fundamental stores of information transmissible from the ancestry to the progeny, the molecular succession, which ensures the transfer of hereditary messages from the cells of one generation to the next generation, and the verbal legacy as a necessary prerequisite of cultural tradition. ${ }^{1}$

Divergent terminologies direct attention to different pattemings; and finding a logically convincing test, acceptable all around, that can determine whether one such system of terms is superior to its rivals, is often impossible. Yet the slow processes of evolution presumably apply to human societies and their symbolic systems as much as to human bodies, so that when logic cannot decide, survival eventually will. ${ }^{2}$

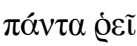

As McNeill points out in his remarkable book on the role of infectious disease in the history of mankind, "one can properly think of most human lives as caught in a precarious equilibrium between the microparasitism of disease organisms and the macroparasitism of large-bodied predators, chief among which have been other human beings". 3 This view, which is abundantly illustrated in the book, leaves several questions open.

Firstly, it is noteworthy that man has surpassed other large-bodied predators like lions and wolves in his ability to command the environment. What was the device that enabled man to achieve higher efficiency in hunting? The obvious answer is: the use of language.

Secondly, it must be noted that man is curiously insensitive as compared with other hunting species. The conjecture that the use of language diminishes the need for direct observation does not explain the rapid disappearance of the hunter's

\footnotetext{
${ }^{1}$ Roman Jakobson, Selected Writings II: Word and Language (The Hague - Paris: Mouton, 1971), 681.

${ }^{2}$ William H. McNeill, Plagues and Peoples (Garden City: Anchor Press/Doubleday, 1976), 8.

${ }^{3}$ Ibidem, 5.
} 
senses. More probably, the faculty of language has an adverse effect on the perceptual capacity of the brain.

Thirdly, the macroparasitism among human beings differs in kind from the relation between predator and prey. Its function is to shorten the food chain. Even cannibalism generally serves a legal purpose and is not merely a way of feeding. Human beings are driven by ideas.

In recent years there has been some debate whether language must be viewed primarily as a means of communication or as a form of self-expression of the human mind. Both of these views start from an anthropocentric conception of language. According to the view advanced in the present article, language is the means of communicating natural forms of self-expression through the human mind.

The influence of concepts is particularly striking in man's economic behaviour. The following example is typical:

What had always seemed commonplace and respectable became, after Veblen, fraudulent, ridiculous and (a favorite word of his) barbaric. This is high art. The American rich never recovered from the sardonic disdain with which Veblen analyzed their behavior. The manners of an entire society were altered as a result. After he made the phrase "conspicuous consumption" a part of the language, the real estate market in Newport was never again the same. What had been the biggest and best was henceforth the most vulgar. "Conspicuous leisure" made it difficult even for the daughters of the rich to relax. Their entertainment had thereafter to be legitimatized by charitable, artistic or even intellectual purpose or, at minimum, sexual relief.

It is hard to think of child labour, war, totalitarianism, or massive unemployment without the driving force of a system of beliefs in conjunction with a blunted sense of perception. The observation that in all Yuman languages the word for 'work' is a loan from Spanish ${ }^{5}$ should be a major blow to any current economic theory.

The force of language is indeed comprehensive. "Even in the most primitive cultures the strategic word is likely to be more powerful than the direct blow". 6 This has little to do with truth or logic because "the normal speaker does not actually feel the clash which the logician requires". 7 The view of language as a tool of the human species is less well-founded than its converse. The question is, in Humpty Dumpty's words, which is to be master.

\footnotetext{
${ }^{4}$ John Kenneth Galbraith, Economics, Peace and Laughter (New York: The New American Library, 1972), $35 \mathrm{f}$.

${ }^{5}$ Werner Winter, personal communication.

${ }^{6}$ Edward Sapir, Selected Writings in Language, Culture and Personality (ed. by David G. Mandelbaum, Berkeley - Los Angeles: University of California Press, 1949), 18.

${ }^{7}$ Ibidem, 27.
} 
The relation of a language to its carrier bears a strong resemblance to that of a parasite to its host. It invades the left hemisphere, diminishing the perceptual capacity of the brain. As a result, man's major capability to change his environment is matched by a minor capability to gain insight from direct observation. An excessive attack may lead to autistic phenomena. If the brain strikes back, it may yield a form of epilepsy. Cruelty is a human characteristic because it results from the substitution by the linguistic parasite of conception for perception.

The formalist philosophy of language, of which generative grammar is but the latest variety, is futile because its subject matter is more easily accessible to the biochemist. The proper subject of the humanities is the behaviour of the linguistic parasite. As in the natural sciences, advance in a humanist discipline springs from observation of what happens under changing circumstances, not from reflection on what is generally known.

Language differs from viral diseases such as measles or smallpox in two respects. First, it is transmitted through sound waves, not through bodily contact. It can therefore be assumed that the fundamental structure of language is much simpler than that of a regular childhood disease. Second, the pattern of mutual adaptation between the individual and his language is much more stable than in the case of a more virulent infection. The process of adjustment alters not only the individual's behaviour and his language, but also the behaviour of the group and the structure of the society at large. The relative ease with which the individual's language responds to a change in the environment and the violent reactions which the use of language provokes among larger groups show that language is ancient in the individual and unfit for the large-sized communities of modern times.

The fast rate of change which language exhibits can be compared with the instability of the influenza virus. It exemplifies a type of change which differs qualitatively from what we are accustomed to regard as the normal type of biological reproduction. In order to clarify the matter I define:

(1) Organic reproduction yields an image which resembles the model to a large extent.

(2) Symbolic reproduction yields an image which resembles the model to a small extent.

Both types of reproduction must be distinguished from physical growth, which changes the size of an object without affecting its internal relationships. A crystal may grow, but does not reproduce itself.

Werner Winter once compared the work of a translator with that of an artist who is asked to create an exact replica of a marble statue, but who cannot secure any marble. This is an apt characterization. Other linguistic activities differ from the translator's work in the absence of the intention to create a replica. They share 
the feature of symbolic reproduction and involve the creation of objects with unforeseen properties.

Logical analysis requires the identifiability of distinguishable elements as belonging to the same set. In the case of an extensional definition, it presupposes a sufficient degree of similarity between the indicated and the intended elements. In the case of an intensional definition it presupposes the applicability of a criterion, which depends on the degree of similarity between the indicated property and the perceptible characteristics of the intended objects. The constructibility of a set is determined by the identifiability of its elements.

Language does not generally satisfy the fundamental requirement of logic. Consider the following example:

(3) Man is numerous.

(4) Socrates is a man.

(5) Socrates is numerous.

From a linguistic point of view, the inference is equivalent to the first syllogism of traditional logic. The point is that a linguistic meaning thrives by virtue of its applications, which cannot be deduced from its implications. The latter must be derived from its applicability, rather than the other way round. Thus, a linguistic meaning has the properties of a non-constructible set.

Now I define:

(6) Existence is the capacity of an element being distinguished.

(7) Truth is the capacity of an element belonging to a set.

(8) Meaning is the capacity of being a set.

(9) The power of a capacity is the set of elements with that capacity.

(10) Symbolization is the power of existence.

(11) Generalization is the power of truth.

(12) Abstraction is the power of meaning.

(13) Mathematics is the study of symbolization.

(14) Logic is the study of generalization.

(15) Philosophy is the study of abstraction.

Thus, a mathematician is typically concerned with the problem of existence, a logician with the problem of truth, and a philosopher with the problem of meaning.

(16) Physics is the phenomenology of existence.

(17) Anthropology is the phenomenology of truth.

(18) Linguistics is the phenomenology of meaning.

The following statement can easily be verified:

(19) Symbolization is a finite simple group of extremely large order. 
- If $x$ can be distinguished and $y$ can be distinguished, then $x$ and $y$ can be distinguished.

- If $x$ and $y$ can be distinguished and $z$ can be distinguished, then $x$ can be distinguished and $y$ and $z$ can be distinguished.

- There is an identity element, viz. nothing, which combines with any distinguishable element without affecting what is distinguished.

- For every distinguishable element $x$ there is an inverse element $x^{-1}$, which is the absence of $x$.

- There is no proper subset of symbolization such that every distinguishable element can be distinguished as an element of the subset.

- The number of distinguishable elements is limited by the finite ability of the senses.

(20) Generalization is the Cartesian product of symbolization and abstraction.

I further define:

(21) Sense is the applicability of meaning.

(22) Formalization is the reduction of meaning to truth.

(23) A contradiction is an element of generalization which is both true and false.

(24) A confusion is an element of abstraction with a contradictory formalization.

The existence of non-constructible sets offers a solution for the problem of the philosopher's stone. I think that the philosopher's stone is a 4-dimensional object and that it is crossing the 3-dimensional surface of a 4-dimensional pond called history. The universe is the slice of the stone which is at the level of the water. The universe originated with a big bang when the stone hit the surface. It is finite and expands as the stone sinks into the water. Why did the stone hit the surface? We shall never know because it is beyond human observation.

The parasitological view of non-constructible sets outlined here has important consequences for the daily practice of human affairs. Language has enabled humanity time and again to discover new techniques, allowing easy exploitation and rapid depletion of hitherto inaccessible resources and thereby renewing or intensifying damage to other forms of life. Its fast rate of change has not permitted a stable, chronic relationship to establish itself. "A stable new disease pattern can arise only when both parties manage to survive their initial encounter and, by suitable biological and cultural adjustments, arrive at a mutually tolerable arrangement. (...) historical experience of later ages suggests that something like 120 to 150 years are needed for human populations to stabilize their response to drastic new 
infections". ${ }^{8}$ Language may be lethal if time does not suffice for humans to adjust to changing conditions.

The reason why we have not been able to locate an extra-terrestrial civilization is probably that it managed to blow itself to pieces before contact could be established: otherwise we would have been blown to pieces ourselves. The survival of the human species requires a strong curtailment of resources available for technological innovation and a large-scale effort to diminish abstraction and to stimulate direct observation by the individual.

[See also 206.]

\footnotetext{
${ }^{8}$ McNeill, o. c., 51.
} 